\title{
Dynamics of High-Speed Precision Geared Rotor Systems
}

\author{
Teik C. Lim ${ }^{1, a}$
}

${ }^{1}$ University of Cincinnati, College of Engineering and Applied Science, 801 Engineering Research Center, Cincinnati, Ohio 45221-0018, USA

\begin{abstract}
Gears are one of the most widely applied precision machine elements in power transmission systems employed in automotive, aerospace, marine, rail and industrial applications because of their reliability, precision, efficiency and versatility. Fundamentally, gears provide a very practical mechanism to transmit motion and mechanical power between two rotating shafts. However, their performance and accuracy are often hampered by tooth failure, vibrations and whine noise. This is most acute in high-speed, high power density geared rotor systems, which is the primary scope of this paper. The present study focuses on the development of a gear pair mathematical model for use to analyze the dynamics of power transmission systems. The theory includes the gear mesh representation derived from results of the quasi-static tooth contact analysis. This proposed gear mesh theory comprising of transmission error, mesh point, mesh stiffness and line-of-action nonlinear, time-varying parameters can be easily incorporated into a variety of transmission system models ranging from the lumped parameter type to detailed finite element representation. The gear dynamic analysis performed led to the discovery of the out-of-phase gear pair torsion modes that are responsible for much of the mechanical problems seen in gearing applications. The paper concludes with a discussion on effectual design approaches to minimize the influence of gear dynamics and to mitigate gear failure in practical power transmission systems.
\end{abstract}

\section{Introduction}

Gears have existed for many decades and played a significant role in powering the industrial revolution. Even though gears are classical machine elements, today, they are still one of the most widely applied mechanical devices in power transmissions employed in automotive, aerospace, marine, rail and industrial applications because of their reliability, precision, efficiency and versatility. In principle, gears provide an efficient and precise means to transmit mechanical motion and power from one rotating shaft to another in a controlled manner. This is accomplished through the gear tooth engagement process by which the driving teeth of the input gear engage and thrust the driven teeth of the output gear as shown in Fig. 1. That discrete mechanical action

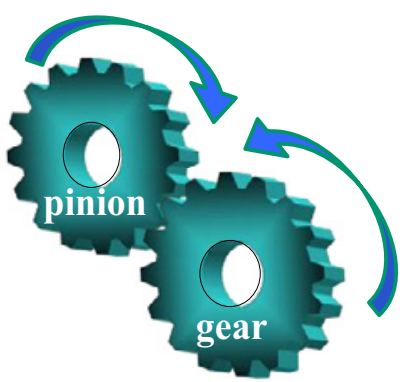

Fig. 1 - A gear pair in action. provides an accurate way to control rotational motion and timing of the power transmissions.

In spite of the extensive use of gears in power transmission systems, their performance and accuracy are often hampered by tooth failure, vibrations and whine noise [1-3], especially in highspeed, high power density geared rotor systems typically used in automotive, aerospace, marine, rail and industrial applications. Most of these mechanical failures and excessive responses are attributed

\section{a Teik.Lim@uc.edu}

This is an Open Access article distributed under the terms of the Creative Commons Attribution License 2.0, which permits unrestricted use, distribution, and reproduction in any medium, provided the original work is properly cited. 
to uncontrollable dynamics of the gear pairs. Therefore, in this study, an attempt is made to understand in a comprehensive manner the source of these failures and to develop effective solutions to mitigate the problems by developing an accurate gear pair dynamic model that contains the essential features capable of explaining the root cause of undesirable gear behaviors.

Early studies [4-6] of gear meshing reveals that the primary excitation in gearing mechanism is parametric in nature due to tooth elastic deformation, profile micro-errors due to manufacturing and design imperfections, and misalignment from the assembly process. These faults and deformations result in micro deviations of the tooth positions or gear rotations relative to its ideal or design state known as the gear transmission error [6-7] as described in Fig. 2. The interaction between the system dynamics and transmission errors produces the dynamic gear mesh force $\mathrm{F}_{\mathrm{m}}$. This force is regarded as the main exciter of gear dynamics. Further explanations are given in latter part of this paper.

Much of the focus of past studies by gear researchers have been mostly devoted towards eliminating transmission errors through improvements in gear tooth form design, and manufacturing and assembly accuracies [8-9]. This strategy also includes profile modifications to readjust the positions of the gear teeth so that they are

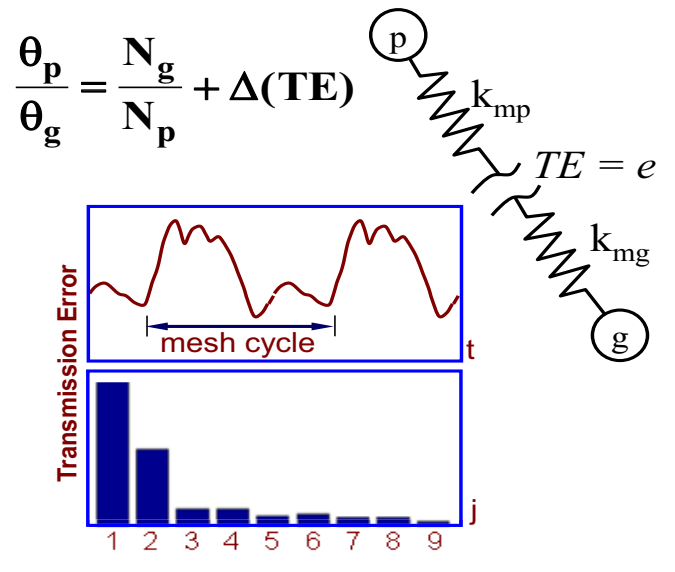

Fig. 2 - Gear transmission error. as close as possible to the ideal or design state in the attempt to minimize transmission error. While this solution has worked in some designs, there are still many applications in which the driveline system dynamics is exceedingly sensitive to transmission error causing even the slightest occurrence of transmission to produce excessively high mesh force excitation. In these cases, simply reducing transmission errors does not eliminate the failure problems. In other cases, the gear excitations are caused by other sources such as mesh stiffness variations, shuttling forces, friction forces, and air/lubricant entrainment [10]. Both scenarios cannot be tackled successfully by merely designing and making high quality gears. The approach proposed in this study by de-sensitizing the driveline system dynamics to gear excitation is believed to produce a more robust power transmission system design than solely making high quality gears. The remaining portion of this paper is devoted to the modeling gear pair dynamics and also understanding the root cause of gear dynamics.

\section{Gear Pair Dynamics}

A generic gear pair system can be manifested as a pair of rotating disk coupled by an infinitesimal spring-damper element as shown in Fig. 3. The position and orientation of the spring-damper and transmission error $e$ represents the gear mesh model. This two degrees of freedom system can be represented mathematically by the following set of equations of motion,

$$
\begin{aligned}
& I_{p} \ddot{\theta}_{p}+\lambda_{p} c_{m}(\dot{\delta}-\dot{e})+\lambda_{p} k_{m} f(\delta-e)=T_{p} \\
& I_{g}^{p} \ddot{\theta}_{g}-\lambda_{g}^{p} c_{m}(\dot{\delta}-\dot{e})-\lambda_{g} k_{m}^{m} f(\delta-e)=-T_{g} \\
& \delta=\lambda_{p} \theta_{p}-\lambda_{g} \theta_{g}
\end{aligned}
$$

where $\lambda$ is the rotational radius. Since this is semi-definite system, the dynamical equations can be deduced into a definite expression assuming $p=\delta-e$ for the mesh deformation as shown in Fig. 3 . The final set of one degree of freedom system is given by,

$$
m_{e} \ddot{p}+c_{m} \dot{p}+k_{m} f(p)=m_{e}\left(\frac{\lambda_{p} T_{p}}{I_{p}}+\frac{\lambda_{g} T_{g}}{I_{g}}-\ddot{e}\right), \quad m_{e}=1 /\left(\lambda_{p}^{2} / I_{p}+\lambda_{g}^{2} / I_{g}\right)
$$



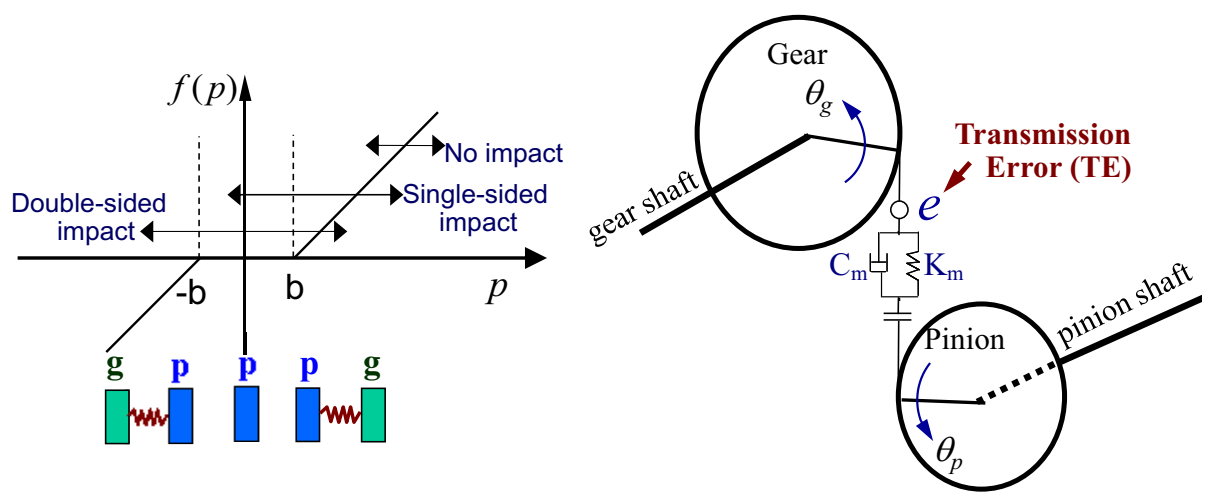

Fig. 3 - Gear pair model with mesh representation.

The modal solution of the above Eq. (4) yields the out-of-phase gear pair torsion mode as shown in Fig. 4. Also, in Fig. 4, the in-phase gear pair torsion mode is shown at $0 \mathrm{~Hz}$ representing the rigid body rotation of the coupled disks, which controls the fundamental transmission of mechanical motion and power. On the other hand, the out-of-phase gear pair torsion mode not only can degrade the fundamental power transmission function but is believed to be the underlying source of gear mesh force excitation. This mode is the primary interest here because it is believed to be responsible for gear tooth failure, vibrations and whine noise.

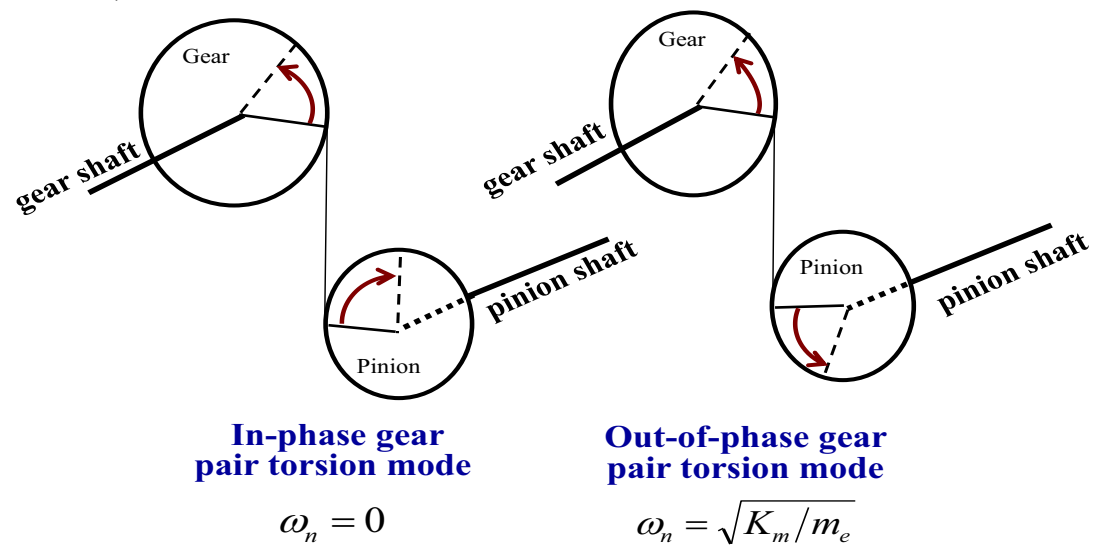

Fig. 4 - The out-of-phase gear pair torsion mode is the primary cause of gear response due to transmission error.

In more complex systems with higher number of coordinates, this one out-of-phase gear pair torsion mode spawn into a class of out-of-phase gear pair torsion modes by coupling with dynamic flexibilities in the rest of the driveline system. This is demonstrated in the next.

\section{System Response}

The geared system dynamic characteristics can be depicted in Fig. 5. As noted above, the gear transmission error interacts with the powertrain system dynamics $D_{m}$ to generate the dynamic mesh force $F_{m}(\omega)=D_{m}(\omega)^{*} e$. An example plot of $F_{m}(\omega)$ is given in Fig. 6. Here, it may be pointed out that the response peaks are all attributed to the out-of-phase gear pair torsion modes described earlier. The dynamic mesh force then excites the attached shaft-bearing assembly. The resultant bearing dynamic forces then excites the gearbox casing and support structures. The vibration of the attached structures in turn radiates noise into the surrounding space. Symbolically, the response $P(\omega)=\Sigma_{r}\left[F_{m}(\omega) * T_{r}(\omega)\right]$ where $\Sigma_{r}$ is the summation over all the vibration transmissibility $T_{r}(\omega)$ through each structural path denoted by subscript $r$ associated with the gearbox. 


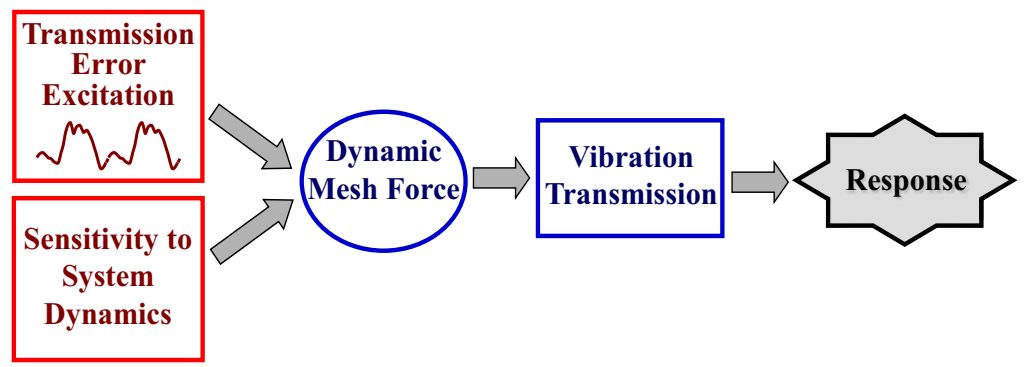

Fig. 5 - Geared rotor system dynamic characteristics.

Firstly, the dynamic mesh force that defines the severity of tooth contact can directly cause degradation in the gearing performance and gear tooth failure as well. Secondly, the excessive vibrations generated throughout the gearbox systems may also cause premature structural failure and high level of whine noise causing annoyance. Thirdly, attempt to increase the operating speed and power may be limited by the vibration and whine noise levels.

\section{Design Guidelines}

From the above theories, it is clear that reducing gear response can be accomplished by designing a small transmission error $e$, de-sensitizing system

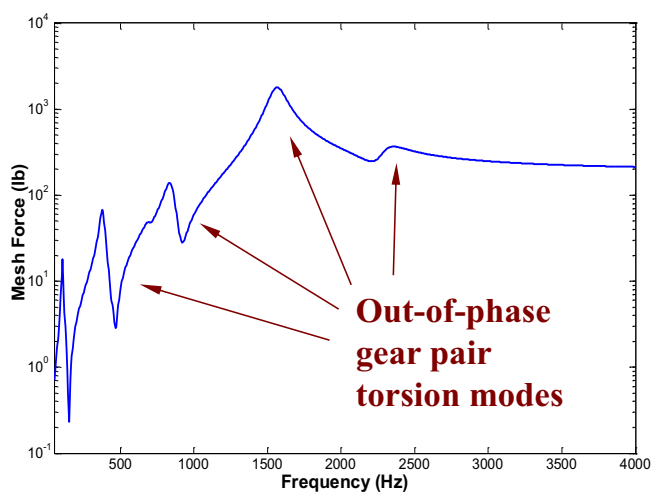

Fig. 6-Dynamic mesh force. dynamics to transmission or lowering $D_{m}$ or minimizing vibration transmissibility $T_{r}$. While the design of smaller transmission error can be performed in isolation, the design of system dynamics $D_{m}$ and vibration transmissibility $T_{r}$ cannot be done separately since both are intertwined due to the fact that their behaviors depend on the same structural components and machine elements.

To control the generation of dynamic mesh force $F_{m}$, a gear mesh compliance theory is developed as summarized in Fig. 7. This provides an alternate way to compute $F_{m}$ based on the upstream (driving pinion side) and downstream (driven gear side) compliances, namely $C_{p}$ and $C_{g}$ for pinion and gear respectively. Using this formulation, $F_{m}=\left[C_{p}+C_{g}+\left(1 / K_{m}\right)\right]^{-1}$, one can easily see that when $C_{p}=-\left[C_{g}+\left(1 / K_{m}\right)\right]$, the dynamic mesh force $F_{m}$ is maximum. In other words, the two compliance functions cross each other with out-of-phase. Therefore, avoiding the out-of-phase crossing events reduce the chances of producing response peaks. Furthermore, if the compliance of one side is far greater than the

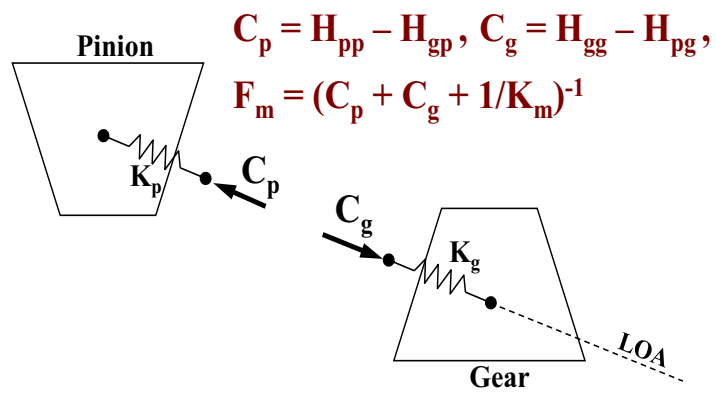

Fig. 7 - Gear mesh compliance theory. other, for example $\left|C_{p}\right|>>\left|C_{g}+\left(1 / K_{m}\right)\right|$ implying $F_{m} \approx\left(1 / C_{p}\right)$, then the side with the greater compliance can be designed to control the dynamic mesh force.

In addition, reducing vibration transmissibility can also lead to improvement in gear response. The issue is that the designs of $F_{m}$ and $T_{r}$ through structural modifications yield opposing effect. Here is why. To reduce gear response, reducing $F_{m}$ and $T_{r}$ is desirable. However, most structural modifications do not lead to reduction in both $F_{m}$ and $T_{r}$. Instead, any reduction in $F_{m}$ usually leads to 
an increase in $T_{r}$ and vice-versa. That means design trade-off must be done in order to find the most optimal solution.

To demonstrate the application of the gear mesh compliance theory and vibration transmissibility, a case study involving the re-design of a motor vehicle driveline to tackle differential gear whine is shown in Fig. 8. Using a substructure-based vehicle system model in which the differential hypoid gear pair is modeled using the above method, the remaining driveline is constructed from finite elements, and the vehicle body is represented with measured transfer functions, a series of parametric studies led to a set of design changes that make the driveline more compliant torsionally, lower ring gear inertia, and tune the final drive carrier mount stiffness. The resultant whine response inside the passenger compartment is substantially lower than the original design.
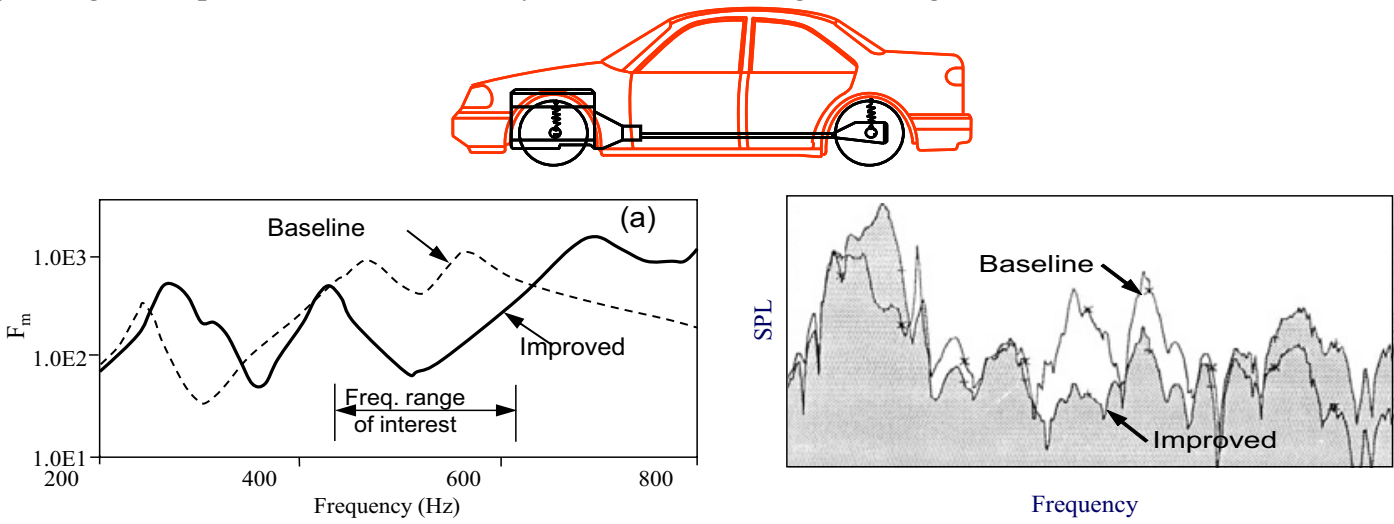

Fig. 8 - Case study on the reduction in the final drive gear mesh force and subsequently sound pressure level (SPL) due to driveline design modifications.

\section{Conclusions}

The present study discusses the discovery of the out-of-phase gear pair torsion modes responsible for excessive gear vibration and whine noise generated in geared-based power transmission systems. The paper also presents the theory of gear mesh force generation and transmissibility, and as well as methodologies for mitigating excessive gear response. The analysis led to the general guidelines of reducing gear response by:

- Minimizing transmission error through design and manufacturing.

- Increasing system torsional compliance will reduce dynamic mesh force but may indirectly increase vibration transmissibility. Hence, solution involving a design trade-off is usually needed.

- Increasing shaft bending stiffness will reduce vibration transmissibility but may indirectly increase vibration transmissibility.

- Reducing gear inertia may reduce dynamic mesh force but may lead to increase in vibration transmissibility in some unique designs.

- Reducing mounting stiffness will usually lower vibration transmissibility

\section{Acknowledgement}

This work is funded by the gear mesh and dynamics consortium at the University of Cincinnati. The gear consortium is presently supported by numerous international automotive and off-highway companies including Meritor, Dana, Chrysler, Linamar, Liugong, Caterpillar, JohnDeere and Hyundai.

\section{References}

[1] J. D. Smith, Gear Noise and Vibration, 2nd ed., Marcel Dekker, New York, (2003). 
[2] S.P. Radzevich, Dudley's Handbook of Practical Gear Design and Manufacture, 2nd ed., CRC Press, Boca Raton, Florida, (2012).

[3] S. Li and A. Kahraman, Micro-pitting fatigue lives of lubricated point contacts: experiments and model validation, International Journal of Fatigue, 48, pp. 9-18, (2013).

[4] H. N. Ozguven, D. R. Houser, Mathematical models used in gear dynamics - A review, J. Sound and Vib., 121 , pp. 383-411, (1988).

[5] M. Hotait and A. Kahraman, Experiments on the relationship between the dynamic transmission error and the dynamic stress factor of spur gear pairs, Mechanism and Machine Theory, 70, pp. 116-128, (2013).

[6] P. Velex, M. Ajmi, Dynamic tooth loads and quasi-static transmission errors in helical gears Approximate dynamic factor formulae, Mech. and Machine Theory, 42, pp. 1512-1526, (2007).

[7] R. Munro, L. Morrish, D. Palmer, Gear transmission error outside the normal path of contact due to corner and top contact, Proc. Inst. Of Mech. Engineers, Part C: J. of Mechanical Engineering Science, 213, pp. 389-400 (1999).

[8] M.S. Tavakoli, D.R. Houser, Optimum profile modifications for the minimization of static transmission errors of spur gears, J. of Mech., Trans., and Automation, 108, pp. 86-94 (1986).

[9] S. Sundaresan, K. Ishii, D.R. Houser, A procesude using manufacturing variance to design gears with minimum transmission error, J. Mech. Des., 113, pp. 318-324 (1991).

[10] D.R. Houser, R. Singh, Gear Noise Short Course Notes, The Ohio State University, Columbus, (2013). 